\section{The prevalence and clinical characteristics of atopic manifestations in patients with irritable bowel syndrome in a Brazilian urban community}

\author{
Rosa L.S. Soares, \\ Hamilton N. Figueiredo, ${ }^{1}$ \\ Pedro F. Moreira Filho,' Rita F. Oliveira, ${ }^{2}$ \\ Carolina D. Gonçalves, ${ }^{2}$ \\ Angelo J.Q. Micuci, ${ }^{2}$ Bruno A. Parada, ${ }^{2}$ \\ Igor Bromonschenkel, ${ }^{2}$ \\ Caio Camargo Rodrigues \\ 'Internal Medicine Department, Unit \\ Intestinal Research, Faculty of Medicine, \\ Rio de Janeiro, Brazil; \\ ${ }^{2}$ Medical School of Faculty of Medicine, \\ Federal Fluminense, University, Rio de \\ Janeiro, Brazil
}

\section{Abstract}

Irritable bowel syndrome (IBS) is a common chronic disorder characterized by recurrent abdominal pain, bowel movement changes, diarrhea, constipation, or both and affect approximately $10-20 \%$ of the general population. Today, IBS is considered a disorder of dysregulation of the so called brain-gut axis and evidence also suggests that gastrointestinal inflammation may be of great importance in the majority of cases of IBS. In addition to gastrointestinal symptoms, many patients have atopic symptoms. The aim of this study was to study the prevalence of atopic manifestations in volunteers with IBS in a Brazilian urban community. Volunteers over 18 years of age were enrolled and evaluated. The participants were evaluated by a gastroenterologist of the Group of Study of Intestinal Diseases at Hospital Universitario Antonio Pedro. All volunteers were evaluated to determine if patients had symptoms compatible with the diagnosis of IBS based on Rome III criteria. The identification of the atopic manifestations was based in a structured questionnaire for atopy. Three hundred and fifty volunteers were enrolled. Of them, 330 volunteers were evaluated: 78 $(23.6 \%)$ of the volunteers had symptoms compatible with IBS (Group I) and 252 (76.3\%) without symptoms compatible with IBS (Group II). Atopic manifestations were present in 46 (65.3\%) in Group I and 105 (41. 0\%) in Group II $(\mathrm{P}=0.0107,0 \mathrm{R}-2.01$ 95\%CI -1. 20-3. 37). The association between atopic manifestations and the presence of IBS was relevant in patients in this Brazilian urban community. This fact may have implications for diagnosis and treatment of patients with IBS.

\section{Introduction}

Irritable bowel syndrome (IBS) affects 10 $20 \%$ of the general population and is the most common reason for patients to consult a gastroenterologist. It is often accompanied by gastrointestinal intestinal and extra-intestinal symptoms. ${ }^{1-6}$ The extra-intestinal symptoms associated to IBS may be correlated with an increase in seeking medical advice, a worse prognosis, and a reduced quality of life..$^{7-11}$ The high prevalence of extra-intestinal symptoms or comorbidities in IBS patients has led investigators to develop a hypothesis regarding underlying pathophysiological mechanisms of these disorders. ${ }^{12-14}$

IBS has been associated with female gender, psychological distress, food intolerance (reproducible adverse reaction to a specific food or food ingredient which is not immune mediated or psychologically based), enteric infections, and previous abdominal surgery. ${ }^{15-21}$ Today IBS is considered a disorder of dysregulation of the so called brain-gut axis and there is evidence to also suggest that inflammation with the gastrointestinal tract may be of great importance in the majority of IBS patients. ${ }^{22,23}$ Approximately $30 \%$ of patients with IBS identify acute infection as an initial trigger for their chronic intestinal infections. ${ }^{17,18,21}$ These findings led to speculation that the immune system may play a role in the pathogenesis of at least a subpopulation of IBS patients. ${ }^{2427}$

In recent studies, excess prevalence of bronchial hyper-responsiveness has been verified among patients with irritable bowel syndrome. ${ }^{27}$ Tobin et al. reported that adults with such atopic symptoms have a high incidence of IBS, suggesting a link between atopy and IBS. ${ }^{28}$

Studies of atopic symptoms or comorbidities are of great scientific interest. Such studies can contribute to the experimental development of a new pathogenesis of IBS. ${ }^{11-14}$ Assessment and improved understanding of atopic somatic symptoms in IBS patients might allow new sub-groups of IBS patients with special characteristics to be identified and thereby lead to the creation of new therapeutic concepts. ${ }^{12,13}$ Distinguishing atopic IBS from nonatopic IBS could help design more appropriate clinical interventions. ${ }^{11,14,28}$ This paper aims to study the prevalence of atopic manifestations in volunteers with IBS in a Brazilian urban community.

\section{Materials and Methods}

Adult volunteers aged 18 and over, residents in Niterói, RJ, Brazil were enrolled during a 2year period from January 2006 to January 2008. The study was approved by the local eth-
Correspondence: Rosa L.S. Soares, Internal Medicine, Department, Faculty of MedicineFederal Fluminense University, Rua Marques do Paraná 189/1002, Rio de Janeiro 24030-210, Brazil. E-mail: rosaleonora@gmail.com

Key words: irritable bowel syndrome, allergy, atopic manifestations, prevalence.

Contributions: RLSS, PFMF, and HNF designed the research and analyzed the data; RFO, CDG, AJQM, BAP, CCR and IB performed the research. RLSS wrote the paper.

Supported by CNPQ-Brazil, National Council to Development of Research.

Conflict of interest: the authors report no conflicts of interest.

Received for publication: 24 April 2010.

Revision received: 12 June 2010.

Accepted for publication: 5 July 2010 .

This work is licensed under a Creative Commons Attribution 3.0 License (by-nc 3.0).

(C) Copyright R.L.S. Soares et al., 2010

Licensee PAGEPress, Italy

Gastroenterology Insights 2010; 2:e11

doi:10.4081/gi.2010.e11

ical committee (n. CAE 0109025800007) and by our institutional review board (IRB) at the Faculty of Medicine. The volunteers were selected from students and employees of the Federal Fluminense University, Faculty of Medicine, Computer Science and Social Sciences, Niterói, RJ, Brazil. They were recruited through a poster affixed on the door of the Group of Study of Intestinal Diseases (Outpatients Unit), Hospital Universitário Antonio Pedro. This approach was used in order to obtain a population sample that would not consist of ambulatory or hospital patients and that would represent the population in general. The exclusion criteria included: those with the diagnostic suspicion of organic disease of the gastrointestinal tract (including positive stool examination for ova and parasites) and those unable to complete the questionnaire. All participants were provided with an IRB-approved information sheet that described the purpose of the study, the voluntary nature of the study, the study aims, methods, and population, and were given opportunities to ask questions. The volunteers were evaluated in our outpatient's clinic at the University Hospital Antonio Pedro (HUAP). After obtaining informed consent, the volunteers went through a complete clinical evaluation. Diagnosis of IBS was based on the Rome III criteria for IBS. These were recurrent abdominal pain or discomfort at least three days per month in the last three months asso- 
ciated with 2 or more of the following: i) improvement with defecation; ii) onset associated with a change in frequency of stool; iii) onset associated with a change in form (appearance) of stool. The volunteers were also asked questions to identify atopic manifestations (seasonal runny nose, nasal congestion, itchy eyes, asthma, food allergy, reactions that cause skin rash, food intolerance triggering digestive symptoms). A total of 330 volunteers completed the surveys. Twenty volunteers were excluded because they presented the diagnostic suspicion of organic disease of the gastrointestinal tract (including positive stool examination for ova and parasites).

\section{Statistical analysis}

Analysis of categorical data was carried out using $\chi^{2}$ or Fisher's exact test, and continuous data were analyzed using independent sample t-test. Odds ratios (OR) with $95 \%$ confidence intervals (CI) were used to measure the associations between the comorbid conditions. $\mathrm{P}<0.05$ was considered significant. The analysis was performed using SAS version 9.1 (SAS Institute, Inc., Cary, NC, USA).

\section{Results}

Three hundred and fifty volunteers (177 women and 173 men) were enrolled in the study. Twenty volunteers who had diagnostic suspicion of organic disease of the gastrointestinal tract were excluded. A total of 330 volunteers completed the surveys. After evaluation of the 330 volunteers, 78 (23.6\%, mean age $27.6 \pm 8.7$ years, 60 female and 18 male) of them met the diagnostic criteria for IBS (Group I-GI). The other 252 (76.3\%, mean age $32.4 \pm 10$ years, 106 female and 146 male) were healthy without symptoms of IBS (Group II GII). There was no significant difference in the mean age of the 2 groups $(P>0.05)$. We also found that the number of female volunteers with diagnosis of IBS was significantly higher than that of male volunteers $(\mathrm{P}<0.05)$ (Table 1).

Atopic manifestations were present in 46 (65.3\%) volunteers of group I and 105 (41.0\%) in GII. Thirty (65.2\%) of the volunteers with IBS had respiratory atopy (26 seasonal allergic rhinitis and 4 asthma), compared with 65 (61.9\%) (55 seasonal allergic rhinitis and 10 asthma) of volunteers without IBS. In addition, 16 volunteers with IBS (34.7\%) had allergic skin manifestations compared with 45 (42.8\%) volunteers without IBS. The percentage of atopic manifestations in volunteers with IBS (GI) was significantly higher than that of GII ( $\mathrm{P}=0.0107$, 0R-2.01 95\%CI -1, 20-3, 37). Twenty-nine (37.1\%) volunteers with IBS reported that a particular food or multiple foods caused abdominal cramping, but the food intolerance was not specifically associated with IBS crises in any of them (Table 1).

Sixteen (76.1\%) of the 21 volunteers with IBS diarrhea predominant (D-IBS) and 11 (73.3\%) of the 15 IBS constipation predominance (C IBS) volunteers had atopic manifestations. Seven (70\%) of the 10 mixed IBS (mIBS) volunteers had allergic complaints. The presence of atopic manifestations was similar between IBS sub-type groups $(\mathrm{P}>0.05)$.

\section{Discussion}

Irritable bowel syndrome is a common disorder worldwide and estimates of prevalence ranged from $9-22 \%$ depending upon the population group studied. The prevalence of symptoms consistent with IBS in the present study, the female predominance and mean age were similar to that reported in the literature. IBS appears to be equally common in the Third World, and we suggest that it can be considered a transcultural functional disorder. ${ }^{1,2,4,6,15}$

In the present study, we investigated the prevalence of atopic manifestations in volunteer non-patients with IBS. We have shown that volunteers with IBS had a higher percentage of allergic symptoms when compared with those without IBS. This finding suggests that there is an association between IBS and allergy history.

The exact IBS pathophysiology remains unknown and the role of allergic reactions in the pathophysiology of IBS remains controversial. ${ }^{21,23,24}$ However, recent interest has been directed to the possible participation of intestinal inflammatory mediators in the pathophysiology of IBS. Mucosal abnormalities such as colonic mastocytosis have been reported in some recent studies..$^{19,21,22}$

Tobin et al. ${ }^{27}$ reported that adults with atopic symptoms report a high incidence of IBS suggesting a link between atopy and IBS. They proposed a new IBS subgroup of patients with atopic symtoms. Our results confirmed the association between IBS and atopic disorders in our study population.

IBS is diagnosed by symptomatic criteria rather than biological markers. Clinical subtypes of IBS are based on the predominant symptoms, such as diarrhea predominant (DIBS) IBS, constipation predominant IBS (C IBS) and mixed IBS (M IBS), ${ }^{4,29}$

Dunlop et al. ${ }^{29}$ reported a significant correlation among atopy and increased intestinal permeability in a subset of IBS patients. We showed in a previous study that the number of positive responses to a skin prick test for food antigens was significantly greater in a D-IBS group of patients than in the other two groups (C IBS and M IBS). ${ }^{30}$ The report of a significant correlation among atopy and increased intestinal permeability suggests that the immune system may play a role in IBS pathogenesis of at least a subpopulation of IBS patients..$^{30-33}$

In the current study, we didn't find a significant association between personal history of allergies and IBS clinical sub-type, but the present results confirm the association between allergy and IBS. ${ }^{23}$

Twenty-nine volunteers with IBS reported intolerance to multiple foods that caused abdominal cramping, but the food intolerance was not specifically associated with IBS crises in any of them. We could not find evidence that suggests a correlation between IBS crises and specific food intolerance in these subjects. This subjective report of adverse food reaction could represent a non-allergic immunological reaction. In a previous study, ${ }^{24}$ we reported that volunteers with a diagnosis of IBS reported higher reactivity to food antigens when compared to patients with functional dyspepsia. We suspect that IBS causes the food sensitivity rather than food sensitivity causes IBS. It is still unclear whether diet is a key factor in exacerbating IBS symptoms and whether dietary manipulation is a valid treatment. ${ }^{3439}$

Recent studies reported a high prevalence of bronchial hyper-responsiveness among patients with irritable bowel syndrome. The

Table 1. Sex predominance, mean age and personal history of allergies in irritable bowel syndrome volunteers and controls.

\begin{tabular}{lccc} 
Characteristics & $\begin{array}{c}\text { BS-GI } \\
(\mathrm{n}=78)(23.6 \%))\end{array}$ & $\begin{array}{c}\text { Controls-GII } \\
(\mathrm{n}=252)(76.3 \%)\end{array}$ & $\mathrm{P}$ \\
Sex, female & 60 female & 106 female & $\mathrm{P}<0.05^{*}$ \\
Mean age (SD) & $(77 \%)$ & $(42 \%)$ & $\mathrm{P}>0.05$ \\
\hline Personal history of allergies & $27.6 \pm 8.7$ years & $32.4 \pm 10$ years & $\mathrm{P}<0.05^{*}$ \\
Respiratory allergy & 46 & 105 & $\mathrm{P}>0.05$ \\
Cutaneous allergy & $(65.3 \%)$ & $(41 \%)$ & $\mathrm{P}>0.05$ \\
& 30 & 65 & \\
\hline *All statistical hypotheses were tested at 0.05 level of significance, and $\mathrm{P}<0.05$ was considered significant. &
\end{tabular}


association between IBS and asthma has also been reported by $0 z o l$ et $a l .{ }^{40}$ These investigators hypothesized a shared respiratory and gastrointestinal pathopyisiological mechanism which produced smooth muscle hyperactivity. In our study, the number of IBS volunteers with asthma was not significantly higher than that of volunteers without IBS.

In summary, the present results confirm the association between allergy and IBS and add new information about the possible participation of intestinal inflammatory mediators in the pathophysiology of IBS. ${ }^{17,20-22,34,35,39,40}$ Further studies are needed to clarify this association. The identification of IBS patients with different clinical sub-types could improve therapeutic options and prevention strategies of IBS.

\section{References}

1. Saito YA, Schoenfeld P, Locke GR 3rd. The epidemiology of irritable bowel syndrome in North America: a systematic review. Am J Gastroenterol 2002;97:1910-5.

2. Talley NJ, Zinsmeister HR, Mellton LJ. Irritable bowel syndrome in a community: symptoms subgroups, risk factors, and health care utilization. Am J Epidemiology 1995;142:76-83.

3 Jones R, Lydeard S. Irritable bowel syndrome in the general population. BMJ 1992;304:87-90.

4. Drossman DA, Camilleri M, Mayer EA, et al. AGA technical review on irritable bowel syndrome. Gastroenterology 2002;123: 2108-31.

5. Quigley EM, Bytzer P, Jones R, et al. Irritable bowel syndrome: the burden and unmet needs in Europe. Dig Liver Dis 2006;38:717-23.

6. Soares RLS, Santos JM, Rocha VRSR. Prevalence of irritable bowel syndrome in a Brazilian Amazon community. Neurogastroenterol Motil 2005;17:883.

7. Maxion-Bergemann S, Thielecke F, Abel F, et al. Costs of irritable bowel syndrome in the UK and US. Pharmacoeconomics 2006;24:21-37.

8. Gralnek IM, Hays RD, Kilbourne A, et al. The impact of irritable bowel syndrome on health-related quality of life. Gastro enterology 2000;119:654-60.

9. Talley NJ, Gabriel SE, Harmsen WS, et al. Medical costs in community subjects with irritable bowel syndrome. Gastroenterology 1995;109:1736-41.

10. Whitehead WE, Olafur P, Jones KR. Systematic review of the comorbidity of irritable bowel syndrome with other disorders: what are the causes and implica- tions? Gastroenterology 2002;122:1140-56.

11. Vandvik PO, Wilhelmsen I, Ihlebaek C, et al. Comorbidity of irritable bowel syndrome in general practice: a striking feature with clinical implications. Aliment Pharmacol Ther 2004;20:1195-203.

12. Riedel A, Schmidtmann M, Stengel A, et al. Somatic comorbidities of irritable bowel syndrome: a systematic analysis. J Psychosom Res 2008;64:573-82.

13. Bonavita V, De Simone R. Towards a definition of comorbidity in the light of clinical complexity. Neurol Sci 2008;29:S99-S102.

14. Toner BB, Akman D. Gender role and irritable bowel syndrome: literature review and hypothesis. Am J Gastroenterol 2000;95: 11-16.

15. Mayer EA, Collins SM. Evolving pathophysiologic models of functional gastrointestinal disorders. Gastroenterology 2002;122: 2032-48.

16. Neal KR, Barker L, Spiller RC. Prognosis in post-infective irritable bowel syndrome: a six year follow-up study. Gut 2002;51:4103.

17. Mckeow N, Parry SD, Stansfield JR, et al. Ons IBS may occur after non-gastrointestinal and intestinal infection. Neurogastroenterol. Motil 2006;18:839-43.

18. McLean PG, Calver AR, Alpers DH, et al. The emerging role of the microbial-gastrointestinal neural axis. Gastroenterology Insights 2009;1:e3:7-11.

19. Barbara G, DeGiorgio R, Stranghellini V, et al. New pathophysiological mechanisms in irritable bowel syndrome. Aliment Pharmacol Ther 2004;20(Suppl.):1-9.

20. Mach T. The brain-gut axis in irritable bowel syndrome - clinical aspects. Med Sci Monit 2004;10:RA125-31.

21. Collins SM. Is the irritable gut an inflamed gut? Scand J Gastroenterol 1992;Suppl 192;102-5.

22. Dainese R, Galliani EA, Lazzarini F, et al. Discrepance between reported food intolerance and sensitization test findings in IBS patients. Am J Gastroenterol 1999;94: 1892-7.

23. Soares RLS, Figueiredo HN, Maneschy C, et al. Correlation between symptoms of the irritable bowel syndrome and the response to the food extract skin pricktest. Braz J Med Biol Res 2004;37:659-62.

24. Yazar A, Atis S, Konca K, et al. Respiratory symptoms and pulmonary functional changes in patients with IBS. Am J Gastroenterol 2001;96:1511-6.

25. Kennedy TM, Jones RH, Hungin AP, et al. Irritable bowel syndrome, gastrooeso phageal reflux, and bronchial hyperresponsiveness in the general population. Gut 1998;43:770-4.

26. Jun Dw, Oy L, Yomm Hj, et al. Bronchial hyperrresponiveness in IBS. Dig Dis Sci 2005;50:1688-91.

27. Tobin M C, Moparty B, Farhadi A, et al. Atopic irritable bowel syndrome: a novelsubgroup of irritable bowel syndrome with allergic manifestations. Ann Allergy Asthma Immunol 2008;100:49-53.

28. Drossman DA, Corazziari E, Talley NJ, et al. Rome III-A multinational consensus document on functional gastrointestinal disorders. Gastroenterology 2006;130: 1480-91.

29. Dunlop SP, Hebden J, Naesdal J, et al. Abnormal intestinal permeability in subgroups of diarrhea predominant IBS. Am J Gastroenterol 2006;101:1288-94.

30. Camilleri M, Gorman H. Intestinal permeability and IBS. Neurogastroenterol Motil 2007;19:545-52.

31. Soares RLS, Figueiredo HN, Santos JM, et al. Discrepancies between the responses to skin prick test to food and respiratory antigens in two subtypes of patients with irritable bowel syndrome. World J Gastroenterol 2008;14:3044-8.

32. Barbara G. Mucosal barrier defects in irritable bowel syndrome. Who left the door open? Am J Gastroenterol 2006;101:12958.

33. Jun DW, Lee Oy, Yoon HJ, et al. Food intolerance and skin prick test in treated and untreated irritable bowel syndrome. World J Gastroenterol 2006;12:2382-7.

34. Zar S, Benson MJ, Kumar D. Food. Specific serum IgG4 and IgE titers to common food antigens in irritable bowel syndrome. Am J Gastroenterol 2005;100:1550-7.

35. Zuo XL, Li YQ, LI WJ, et al. Alterations of food antigen-specific serum immunoglobulins $\mathbf{G}$ and $\mathrm{E}$ antibodies in patients with irritable bowel syndrome and functional dyspepsia. Clin Exp Allergy 2007;37:82330.

36. Locke GR 3rd, Zinsmeister AR, Talley NJ, et al. Risk factors for irritable bowel syndrome: role of analgesics and food sensitivities. Am J Gastroenterol 2000;95:15765.

37. Jones VA, McLaughan P, Shorthouse M, et al. Food intolerance: a major factor in the pathogenesis of irritable bowel syndrome. Lancet 1982;2:1115-7.

38. Zwetchkenbaum J, Burakoff R. Irritable bowel syndrome and hypersensitivity. Ann Allergy 1988;61:47-9

39. Ozol D, Uz E, Bozalan R, et al. Relationship between asthma and Irritable bowel syndrome: role of food allergy. Journal of Asthma 2006;43:773-5.

40. Camilleri M. Treating irritable bowel syndrome: overview, perspective and future therapies. Br J Pharmacol 2004;141:123748. 